\title{
SOME REMARKS ON RESTRICTION OF THE FOURIER TRANSFORM FOR GENERAL MEASURES
}

\author{
Per Sjölin And Fernando Soria
}

\begin{abstract}
In this paper we establish a formal connection between the average decay of the Fourier transform of functions with respect to a given measure and the Hausdorff behavior of that measure. We also present a generalization of the classical restriction theorem of Stein and Tomas replacing the sphere with sets of prefixed Hausdorff dimension $n-1+\alpha$, with $0<\alpha<1$.
\end{abstract}

\section{Introduction}

Let $\nu$ be a positive, finite Borel measure in $\mathbf{R}^{n}, n \geq 1$. Assume also that $\nu$ has compact support. We define for a given $f \in L^{1}\left(\mathbf{R}^{n}\right)$ and for $R>0$ the $R$-average of the Fourier transform of $f$ with respect to the measure $\nu$ as

$$
\sigma(f)(R)=\sigma_{\nu}(f)(R)=\int|\widehat{f}(R \xi)|^{2} d \nu(\xi) .
$$

In this work we shall study the following problem: Assuming that $0<\alpha \leq n$, for which values of $\beta$ does there exist a constant $C=C_{\alpha, \beta}$ such that the estimate

$$
\sigma(f)(R) \leq \frac{C}{R^{\beta}} \int_{\mathbf{R}^{n}}|\widehat{f}(\xi)|^{2} \frac{d \xi}{|\xi|^{n-\alpha}}, \quad R>1
$$

holds for all $f$ in, say, $\mathcal{C}_{c}^{\infty}\left(\mathbf{R}^{n}\right)$ with supp $f \subset B_{1}$ ? (Here $B_{r}$ denotes the ball of radius $r>0$ centered at the origin.)

Research partially supported by the European Commission, TMR network "Harmonic Analysis" and by the Göran Gustafsson Foundation. 
Estimates of this sort have been considered in $[\mathbf{5}],[\mathbf{1}],[\mathbf{9}]$ and $[\mathbf{1 0}]$ for the case $d \nu=d x_{\mid \mathbf{S}^{n-1}}=d \omega$, the Lebesgue measure on the unit sphere (see also [4]). In these papers the authors study the minimum Hausdorff dimension that a set $A$ must have to ensure that the "distance set" $\{|x-y|: x, y \in A\}$ has positive measure on $\mathbf{R}_{+}$. For that, they have to look at the problem only for the case in which $f(x) \geq 0$. This is of significant importance as we will see below.

In [2] and [3] a similar problem is considered for $d \nu=d \omega$ again, and for $\beta=n-1$. However, the right hand side of (1), i.e., the norm of $f$ in the Sobolev space $\dot{H}^{\frac{\alpha-n}{2}}$ is replaced by a larger one. In fact, (1) cannot hold for any value of $\alpha$ if we insist in asking $\beta$ to be $n-1$, except when $\alpha=n$ (see Theorem 3).

The results of our work, as most of the papers mentioned above, describe some aspects of the behavior of the Fourier transform when restricted to lower dimensional sets. In the classical theory, the curvature of the sets plays a central role. We study here a slightly different problem, the average decay of the restriction with respect to arbitrary measures, and, as we will see, it is the geometry given by the Hausdorff behavior of our sets what really matters in this case. Also, in the last section we investigate a generalization of the usual restriction theorems replacing the sphere with sets of prefixed Hausdorff dimension $n-1+\alpha$, with $0<\alpha<1$.

\section{Main results}

In the following theorems we shall use the condition

$$
\nu\left(B_{t}\left(x_{0}\right)\right) \leq C t^{\gamma}, \quad t>0, \quad x_{0} \in \mathbf{R}^{n},
$$

where $\gamma \geq 0$ and $B_{t}\left(x_{0}\right)$ denotes the ball $\left\{x:\left|x-x_{0}\right|<t\right\}$.

Theorem 1. If (2) holds then (1) holds with $\beta=\alpha+\gamma-n$ for all $f$ of the above type and $0<\alpha \leq n$.

As we will see below, this theorem is sharp for certain measures, like the Lebesgue measure associated to the unit sphere. In general we have the following converse statement

Theorem 2. Assume that (1) holds for all $f$ of the above type and for some $\beta \geq 0$ and some $\alpha, 0<\alpha \leq n$. Then (2) holds with $\gamma=\beta$. 
Proof of Theorem 1: Let us observe that

$$
\begin{aligned}
\sigma(f)(R) & =\int_{\mathbf{R}^{n}} \widehat{f}(R \xi) \overline{\widehat{f}(R \xi)} d \nu(\xi) \\
& =\int_{\mathbf{R}^{n}} \int_{\mathbf{R}^{n}}\left(\int_{\mathbf{R}^{n}} e^{-i R \xi \cdot(x-y)} d \nu(\xi)\right) f(x) \overline{f(y)} d x d y \\
& =\int_{\mathbf{R}^{n}} \int_{\mathbf{R}^{n}} \widehat{\nu}(R(x-y)) f(x) \overline{f(y)} d x d y .
\end{aligned}
$$

Choose now $\phi \in \mathcal{C}_{c}^{\infty}\left(\mathbf{R}^{n}\right)$ so that $\phi(x)=1$ for $|x| \leq 2$ and with $\phi$ even. Then, using the support condition on $f$ we have

$$
\begin{aligned}
\sigma(f)(R) & =\int_{\mathbf{R}^{n}} \int_{\mathbf{R}^{n}} \widehat{\nu}(R(x-y)) \phi(x-y) f(x) \overline{f(y)} d x d y \\
& =\int_{\mathbf{R}^{n}} \int_{\mathbf{R}^{n}} K_{R}(y-x) f(x) \overline{f(y)} d x d y \\
& =\int_{\mathbf{R}^{n}} K_{R} * f(y) \overline{f(y)} d y,
\end{aligned}
$$

where $K_{R}(x)=\widehat{\nu}(-R x) \phi(x)$. Hence, by Plancherel

$$
\sigma(f)(R)=c \int_{\mathbf{R}^{n}} \widehat{K}_{R}(\xi) \widehat{f}(\xi) \widehat{\hat{f}(\xi)} d \xi
$$

and, therefore, suffices to prove

$$
\left|\widehat{K}_{R}(\xi)\right| \leq \frac{C}{R^{\beta}} \frac{1}{|\xi|^{n-\alpha}}
$$

with $\beta=\alpha+\gamma-n$.

To do this observe that

$$
\widehat{K}_{R}(\xi)=\int \widehat{\phi}(\xi-R y) d \nu(y) .
$$

Now, if $|\xi| \geq A R$ (for $A$ large enough depending on the diameter of the support of $\nu$ ) then $\left|\widehat{K}_{R}(\xi)\right| \leq C_{N}|\xi|^{-N}$ for any positive integer $N$. This follows easily from the fact that $\widehat{\phi}$ is a Schwartz function. Thus (3) holds in this case. It remains to study the case $|\xi| \leq A R$. For that we use 
condition (2):

$$
\begin{aligned}
\left|\widehat{K}_{R}(\xi)\right| & \leq C_{N} \int \frac{1}{1+|\xi-R y|^{N}} d \nu(y) \\
& \leq C_{N}\left(\int_{|\xi-R y|<1} d \nu(y)+\sum_{k=1}^{\infty} \int_{2^{k-1} \leq|\xi-R y|<2^{k}} 2^{-k N} d \nu(y)\right) \\
& \leq C_{N} \sum_{k=0}^{\infty} 2^{-k N} \nu\left(B_{\frac{2 k}{R}}(\xi / R)\right) \\
& \leq C \sum_{k=0}^{\infty} 2^{-k N}\left(\frac{2^{k}}{R}\right)^{\gamma} \leq C R^{-\gamma},
\end{aligned}
$$

for sufficiently big $N$. Now (3) follows if $R^{-\gamma} \leq C R^{-\beta}|\xi|^{\alpha-n}$. That is, if $|\xi|^{n-\alpha} \leq C R^{\gamma-\beta}$. But this holds since $n-\alpha=\gamma-\beta \geq 0$ and $|\xi| \leq A R$. This finishes the proof of Theorem 1.

We are thankful to the referee for pointing out the connection between our result and a previous result of Strichartz in [12] where he shows that if the measure $\nu$ has property (2) (what he calls the locally uniformly $\gamma$-dimensional property) then one has

$$
\int_{|x| \leq R}|\widehat{f d \nu}(x)|^{2} d x \leq C R^{n-\gamma}|| f \|_{L^{2}(\nu)}^{2} .
$$

As can be seen readily, this corresponds to the dual statement of our theorem in the special case $\alpha=n$. Originally we were not interested in this end point case. However, after the above observation by the referee and the fact that our arguments trivially covered it, we decided to include it for completeness.

Proof of Theorem 2: Choose $g$ an even function in the Schwartz class $\mathcal{S}$ such that $\operatorname{supp} \widehat{g} \subset B_{\frac{1}{2}}$ and $|g(x)| \geq 1$ on $B_{1}$. Fix $t$, with $0<t<1$, and $x_{0} \in \mathbf{R}^{n}$. Set $R=\frac{1}{t}$ and define $f \in \mathcal{S}$ by $\widehat{f}(R y)=g\left(R\left(y-x_{0}\right)\right)$. It follows that $\widehat{f}(y)=g\left(y-R x_{0}\right)$ and $f(x)=(2 \pi)^{-n} e^{i R x_{0} \cdot x} \widehat{g}(x)$. Now, assuming that (1) holds for some $\alpha$ and some $\beta$ we have

$$
\begin{aligned}
\nu\left(B_{t}\left(x_{0}\right)\right) & \leq \int_{\mathbf{R}^{n}}\left|g\left(R\left(y-x_{0}\right)\right)\right|^{2} d \nu(y) \\
& \leq \frac{C}{R^{\beta}} \int_{\mathbf{R}^{n}}\left|g\left(y-R x_{0}\right)\right|^{2} \frac{d y}{|y|^{n-\alpha}} .
\end{aligned}
$$

We conclude that $\nu\left(B_{t}\left(x_{0}\right)\right) \leq C_{g} t^{\beta}$, since $\left.\int_{\mathbf{R}^{n}} \mid g\left(y-y_{0}\right)\right)\left.\right|^{2}|y|^{\alpha-n} d y$ is bounded by a constant independent of $y_{0}$ whenever $0<\alpha \leq n$. 


\section{Remarks.}

If we restrict ourselves to the case in which the functions are assumed to be positive we could get better estimates for (1) than those obtained in Theorem 1, at least for certain values of $\alpha$. For instance, suppose that we know the rate of decay of the Fourier transform of $d \nu$; that is, suppose that

$$
|\widehat{\nu}(\xi)| \leq \frac{C}{|\xi|^{\frac{\eta}{2}}}
$$

for some $\eta \geq 0$. Then we have the following estimate for every $0<\alpha \leq \frac{\eta}{2}$

$$
\sigma(f)(R) \leq \frac{C}{R^{\alpha}} \int_{\mathbf{R}^{n}}|\widehat{f}(\xi)|^{2} \frac{d \xi}{|\xi|^{n-\alpha}}
$$

provided that $f \geq 0$.

The proof of this fact is straightforward. Write as in Theorem 1

$$
\sigma(f)(R)=\int_{\mathbf{R}^{n}} \int_{\mathbf{R}^{n}} \widehat{\nu}(R(x-y)) f(x) \overline{f(y)} d x d y .
$$

Now, using that $\nu$ is finite and (4) we see that

$$
|\widehat{\nu}(\xi)| \leq C \min \left\{1, \frac{1}{|\xi|^{\frac{\eta}{2}}}\right\} \leq \frac{C}{|\xi|^{\alpha}}
$$

if $\alpha \leq \eta / 2$. Hence, if $f \geq 0$ we conclude

$$
\sigma(f)(R) \leq C \int_{\mathbf{R}^{n}} \int_{\mathbf{R}^{n}} \frac{f(x) f(y)}{(R|x-y|)^{\alpha}} d x d y=\frac{C}{R^{\alpha}} \int_{\mathbf{R}^{n}}|\widehat{f}(\xi)|^{2} \frac{d \xi}{|\xi|^{n-\alpha}} .
$$

The best value of $\eta$ for which (4) holds (the supremum of these values, rather) is sometimes called the Fourier exponent of $\nu$. More precisely, the Fourier dimension of a compact set $E$ is defined as the supremum of the Fourier exponents of all the probability measures supported in $E$. For any smooth hypersuface of $\mathbf{R}^{n}$ with non-vanishing curvature we know that this value is $n-1$. Thus, if we take $d \nu=d \omega$ we have that (1) holds with $\beta=\alpha$ provided that $0<\alpha \leq \frac{n-1}{2}$ and that $f$ is assumed positive. One might think from this that Theorem 1 is far from optimal. However, for "flat" sets, where the Fourier dimension is 0, it gives positive results about the average decay (1). For instance, if we take Lebesgue measure on the surface of the unit cube, then its Fourier exponent is 0 but nevertheless (1) holds with $\beta=\alpha-1$ for every $1<\alpha \leq n$. (This was observed also in $[\mathbf{1 0}]$.) 
Another classical example, now in dimension 1, is provided by the usual Cantor set. As is well known (see the book by P. Mattila [6, p. 168]) this set has Fourier dimension 0, so nothing will be gained in terms of the average decay of $L^{2}$-functions from this. However, the standard measure on the set satisfies (2) with $\gamma=\log 2 / \log 3$ and, therefore, Theorem 1 gives (1) with $\beta=\alpha+\log 2 / \log 3-1$.

Remember that in the above argument it was essential to have $f \geq 0$. In fact, as the next result states, Theorem 1 is sharp in the case $d \nu=d \omega$.

Theorem 3. Fix $0<\alpha \leq n$ and set $\beta(\alpha)=\sup \{\beta:$ (1) holds for all $f$, supp $\left.f \subset B_{1}\right\}$. Then if we consider the usual measure on the unit sphere, $d \nu=d \omega$ we have $\beta(\alpha)=\alpha-1$.

In other words, what Theorem 3 says is that the average properties (1), at least in the two cases considered of the unit sphere and the surface of the unit cube, are determined by the Hausdorff dimension $(n-1$ in this case) rather than by the Fourier exponent of the natural measures associated to them.

Proof of Theorem 3: It is clear that (2) holds with $\gamma=n-1$ and invoking Theorem 1 we conclude that (1) holds with $\beta=\alpha-1$. Hence, $\beta(\alpha) \geq \alpha-1$ (see also Theorem 2 in $[\mathbf{1 0}]$ ). It remains to prove that $\beta(\alpha) \leq \alpha-1$.

Assume that (1) holds and choose $\phi \in \mathcal{C}_{c}^{\infty}(\mathbf{R})$ such that $\operatorname{supp} \phi \subset$ $\left(-\frac{1}{4}, \frac{1}{4}\right)$ and $\widehat{\phi}(0) \neq 0$. Take also $\psi \in \mathcal{C}_{c}^{\infty}\left(\mathbf{R}^{n-1}\right)$ with supp $\psi \subset B_{\frac{1}{2}}$ and $\widehat{\psi}(0) \neq 0$. Given $R>1$ we define $F$ as

$$
F\left(x_{1}, x^{\prime}\right)=e^{i R x_{1}} \phi\left(x_{1}\right) R^{\frac{n-1}{2}} \psi\left(R^{\frac{1}{2}} x^{\prime}\right)
$$

where we denote $x^{\prime}=\left(x_{2}, \ldots, x_{n}\right)$. It follows that

$$
\widehat{F}\left(\xi_{1}, \xi^{\prime}\right)=\widehat{\phi}\left(\xi_{1}-R\right) \widehat{\psi}\left(\xi^{\prime} R^{-\frac{1}{2}}\right)
$$

and, as one can easily see, $\sigma(F)(R) \geq c R^{-\frac{n-1}{2}}$. On the other hand, given $\epsilon>0$ we have

$$
\begin{aligned}
& \int_{\mathbf{R}^{n}}|\widehat{F}(\xi)|^{2} \frac{d \xi}{|\xi|^{n-\alpha}} \\
& =\iint\left|\widehat{\phi}\left(\xi_{1}-R\right)\right|^{2}\left|\widehat{\psi}\left(\xi^{\prime} R^{-\frac{1}{2}}\right)\right|^{2} \frac{1}{|\xi|^{n-\alpha}} d \xi_{1} d \xi^{\prime} \\
& \leq \int_{\left|\xi_{1}-R\right| \leq R^{\epsilon}} \int_{\left|\xi^{\prime}\right| \leq R^{1 / 2+\epsilon}}\left|\widehat{\phi}\left(\xi_{1}-R\right)\right|^{2}\left|\widehat{\psi}\left(\xi^{\prime} R^{-\frac{1}{2}}\right)\right|^{2} \frac{1}{|\xi|^{n-\alpha}} d \xi_{1} d \xi^{\prime}+C R^{-N} \\
& \leq C R^{\frac{n-1}{2}}+\epsilon n+\alpha-n
\end{aligned}
$$


and (1) implies that $R^{-\frac{n-1}{2}} \leq C R^{-\beta} R^{\frac{n-1}{2}+\epsilon n+\alpha-n}$. Hence, since $\epsilon$ can be taken arbitrarily small we obtain $\beta \leq \alpha-1$ and $\beta(\alpha) \leq \alpha-1$. This completes the proof of Theorem 3.

\section{An example of $L^{p}-L^{2}$ restriction.}

Let $n \geq 2$. Recall the classical result of Stein-Tomas about restriction of the Fourier transform to spheres of $\mathbf{R}^{n}$ (see for example [11]) which states that

$$
\left(\int_{\mathbf{S}^{n-1}}|\widehat{f}(\omega)|^{2} d \omega\right)^{1 / 2} \leq C\|f\|_{p}, \quad 1 \leq p \leq \frac{2(n+1)}{n+3} .
$$

On the other hand it is easy to see from the Hausdorff-Young inequality that

$$
\left(\int_{B_{1}}|\widehat{f}(y)|^{2} d y\right)^{1 / 2} \leq C|| f \|_{p}, \quad 1 \leq p \leq 2
$$

We shall here find some estimates which are intermediate to these results. We could do this for instance by means of an interpolating sequence of measures, defining an analytic family of operators and invoking then the interpolation theorem of Stein in this context. An example of this could be given by the family of measures

$$
\mu_{z}=\frac{1}{\Gamma(z)}\left(1-|z|^{2}\right)_{+}^{z-1}
$$

Our example however will be rather on "intermediate sets" between the unit sphere and the unit ball and is more related in spirit to the kind of problems about Hausdorff dimension that we have considered before. More precisely, we shall prove the following

Theorem 4. For every $\alpha$, with $0<\alpha<1$, and for $1<p_{0}<\frac{2(n+1-\alpha)}{n+3-3 \alpha}$ one can find a set $E \subset(0,1]$ with $\operatorname{dim} E=\alpha$ and such that if we define

$$
S=S(E)=\left\{x \in \mathbf{R}^{n}:|x| \in E\right\}
$$

then there exists a natural probability measure $\nu$ on $S$ for which one has

$$
\left(\int_{S}|\widehat{f}(y)|^{2} d \nu(y)\right)^{1 / 2} \leq C\|f\|_{p}, \quad 1 \leq p \leq p_{0} .
$$


We will obtain this as a consequence of results by Mockenhaupt in [7]. For fixed $\alpha, 0<\alpha<1$, and small $\epsilon>0$ the idea is to consider as above a compact subset $E$ of $\left[\frac{1}{2}, 1\right]$ of dimension $\alpha$ with the property that there exists a probability measure $\mu$ supported in $E$ such that

$$
|\widehat{\mu}(\xi)| \leq \frac{C}{|\xi|^{\frac{\alpha}{2}-\epsilon}}
$$

and

$$
\mu\left(B_{t}\left(x_{0}\right)\right) \leq C t^{\alpha} .
$$

A particular construction, using Cantor-like sets and extending previous work of Salem [8], can be found in [7], where the following theorem is also proved

Theorem A. Let $\nu$ be a compactly supported positive measure in $\mathbf{R}^{n}$ which satisfies the estimates

$$
|\widehat{\nu}(\xi)| \leq \frac{C}{|\xi|^{\frac{\eta}{2}}}, \quad \eta>0,
$$

and

$$
\nu\left(B_{t}\left(x_{0}\right)\right) \leq C t^{\gamma}, \quad \gamma>0
$$

Then

$$
\left(\int_{\mathbf{R}^{n}}|\widehat{f}(y)|^{2} d \nu(y)\right)^{1 / 2} \leq C\|f\|_{p}, \quad 1 \leq p<\frac{2(2 n-2 \gamma+\eta)}{4(n-\gamma)+\eta} .
$$

Proof of Theorem 4: Let us consider a set $E$ and a measure $\mu$ satisfying (7) and (8) as above and let us look at the product measure defined on $S(E)$ by $d \nu=c d \mu(r) d \omega$ ( $c$ is just a normalizing constant so that $\nu$ becomes a probability). It is then easy to see

$$
\nu\left(B_{t}\left(x_{0}\right)\right) \leq C t^{n-1+\alpha}, \quad x_{0} \in \mathbf{R}^{n} .
$$

We also have

$$
\begin{aligned}
\widehat{\nu}(\xi)= & c \int_{E}\left(\int_{\mathbf{S}^{n-1}} e^{-i \xi \cdot(s \omega)} d \omega\right) d \mu(s) \\
= & C \int_{E} \mathcal{J}_{\frac{n-2}{2}}(r s)(r s)^{\frac{2-n}{2}} d \mu(s) \\
= & c_{1} \int_{E} e^{i r s}(r s)^{\frac{1}{2}-\frac{n}{2}} d \mu(s)+c_{2} \int_{E} e^{-i r s}(r s)^{\frac{1}{2}-\frac{n}{2}} d \mu(s) \\
& +\mathcal{O}\left(r^{-\frac{n}{2}-\frac{1}{2}}\right) \\
= & \mathcal{O}\left(r^{-\frac{\alpha}{2}+\epsilon-\frac{n}{2}+\frac{1}{2}}\right),
\end{aligned}
$$


where $r=|\xi|$. Here we have used (7) and well known asymptotic formulas for the Bessel function $\mathcal{J}_{\frac{n-2}{2}}$. Therefore, $\nu$ satisfies the hypothesis of the above theorem with exponents $\gamma=n-1+\alpha$ and $\eta=n-1+\alpha-2 \epsilon$. Observing that

$$
\frac{2(2 n-2 \gamma+\eta)}{4(n-\gamma)+\eta}=\frac{2(n+1-\alpha-2 \epsilon)}{n+3-3 \alpha-2 \epsilon}
$$

in our case, we then conclude that

$$
\left(\int_{\mathbf{R}^{n}}|\widehat{f}(y)|^{2} d \nu(y)\right)^{1 / 2} \leq C\|f\|_{p}, \quad 1 \leq p \leq p_{0}
$$

if $p_{0}<\frac{2(n+1-\alpha)}{n+3-3 \alpha}$ by choosing an appropriate, small $\epsilon$.

Acknowledgment. The second author wants to express his gratitude to Anthony Carbery for many enlightening conversations about this subject.

\section{Bibliography}

1. J. Bourgain, Hausdorff dimension and distance sets, Israel J. Math. 87 (1994), 193-201.

2. A. CARBery AND F. Soria, Pointwise Fourier inversion and localisation in $\mathbf{R}^{n}$, J. Fourier Anal. Appl. 3 (1997), 847-858.

3. A. Carbery, F. Soria And A. Vargas, In preparation.

4. K. FAlCONer, On the Hausdorff dimension of distance sets, Mathematika 32 (1985), 206-212.

5. P. Mattila, Spherical averages of Fourier transforms of measures with finite energy; dimension of intersections and distance sets, Mathematika 34 (1987), 207-228.

6. P. Mattila, "Geometry of Sets and Measures in Euclidean Spaces," Cambridge Studies in Advanced Mathematics 44, Cambridge University Press, Cambridge, 1995.

7. G. Mockenhaupt, "Bounds in Lebesgue spaces of oscillatory integral operators," Habilitationsschrift, Siegen, 1996.

8. R. SALEM, On singular monotonic functions whose spectrum has a given Hausdorff dimension, Ark. Mat. 1 (1951), 353-365.

9. P. SJÖLIN, Estimates of spherical averages of Fourier transforms and dimensions of sets, Mathematika 40 (1993), 322-330. 
10. P. SJÖLIN, Estimates of averages of Fourier transforms of measures with finite energy, Ann. Acad. Sci. Fenn. Math. 22 (1997), 227-236.

11. E. M. Stein, "Harmonic Analysis," Princeton Univ. Press, Princeton, NJ, 1993.

12. R. Strichartz, Fourier asymptotics of fractal measures, J. Funct. Anal. 89(1) (1990), 154-187.

13. A. Zygmund, "Trigonometric Series," Cambridge Univ. Press, London-New York, 1968.

\section{Per Sjölin:}

Department of Mathematics

Royal Institute of Technology

S-100 44 Stockholm

SWEDEN

e-mail: pers@math.kth.se
Fernando Soria:

Departamento de Matemáticas

Universidad Autónoma de Madrid

28049 Madrid

SPAIN

e-mail: fernando.soria@uam.es

Primera versió rebuda el 2 de setembre de 1998 , darrera versió rebuda el 27 de juliol de 1999 